\title{
A Kac-potential treatment of nonintegrable interactions
}

\author{
Benjamin P. Vollmayr-Lee ${ }^{1,2}$ and Erik Luijten ${ }^{3}$ \\ ${ }^{1}$ Department of Physics, Bucknell University, Lewisburg, PA 1783\%, USA* \\ ${ }^{2}$ Institut für Physik, WA 331, Johannes Gutenberg-Universität, D-55099 Mainz, Germany \\ ${ }^{3}$ Institute for Physical Science and Technology, University of Maryland, College Park, MD 20742-2431, USA ${ }^{\dagger}$
}

(November 1, 2018)

\begin{abstract}
We consider $d$-dimensional systems with nonintegrable, algebraically decaying pairwise interactions. It is shown that, upon introduction of periodic boundary conditions and a long-distance cutoff in the interaction range, the bulk thermodynamics can be obtained rigorously by means of a Kacpotential treatment, leading to an exact, mean-field-like theory. This explains various numerical results recently obtained for finite systems in the context of "nonextensive thermodynamics," and in passing exposes a strong regulator dependence not discussed in these studies. Our findings imply that, contrary to some claims, Boltzmann-Gibbs statistics are sufficient for a standard description of this class of nonintegrable interactions.
\end{abstract}

PACS numbers: 05.20.Jj, 05.50.+q, 05.70.Fh, 64.60.-i

\section{INTRODUCTION}

In studies of critical phenomena the range of the pairwise interaction that couples the degrees of freedom is an important consideration. For interactions which decay algebraically at large distances, three classes of critical behavior may be obtained. With the standard notation $u(r) \sim-1 / r^{d+\sigma}$, one finds for system dimensionality $d<4$ that the criticality may be characterized as short-range for $\sigma>2-\eta_{\mathrm{sr}}$, non-classically long-range for $d / 2<\sigma<2-\eta_{\mathrm{sr}}$, and classically long-range for $0<\sigma<d / 2$, where $\eta_{\mathrm{sr}}$ is the correlation-function exponent in the corresponding system with short-range interactions [1.22. The critical behavior matches at the bordering cases (e.g., $\sigma \rightarrow d / 2$ from above and below) with additional logarithms. However, for $\sigma \leq 0$ the interactions are nonintegrable, i.e. $\int d^{d} r u(r) \rightarrow \infty$, and so, under standard definitions, the thermodynamic limit does not exist. (See Refs. [3 5] for rigorous treatments.)

Nevertheless, recent studies have focused on this nonintegrable regime, typically using a finite system size to render the total system energy finite. These results are then interpreted as "nonextensive thermodynamics" [6], in which the system energy density scales with some positive power of the system size, as do intensive variables such as temperature. Examples of such works include molecular-dynamics simulations of two- and threedimensional systems with variants of the Lennard-Jones potential [7,8], Monte Carlo simulations of one- and twodimensional Ising [9,10] and Potts [11 systems, and a numerical study of the $X Y$ chain 12. Monte Carlo simulations have also suggested classical critical behavior for a stochastic cellular automaton with long-range in- teractions in the regime $\sigma \leq 0$ [13]. On the basis of these numerical studies of finite systems, several authors have conjectured that "nonextensive criticality" should be classical [9, 11,12$]$.

Here we present an alternate approach to nonintegrable interactions, quite similar to, and in certain cases equivalent to, the use of the Kac potential. We introduce a long-distance cutoff in the interactions at some finite distance $R$, which enables us to use periodic boundary conditions and thus consider homogeneous systems. We then find the energy density in the thermodynamic limit to scale as a power of $R$ rather than of the system size $L$, that is, we maintain extensitivity. By multiplying the pair interaction by the appropriate negative power of $R$ we recover a well-defined $R \rightarrow \infty$ limit. In this way, by using standard methods (including the conventional canonical ensemble), we find an exact solution for the free energy for all $-d \leq \sigma \leq 0$, and so demonstrate the classical nature of the criticality without resort to simulations or conjecture! These results have been announced previously in Ref. [14].

Indeed, this is not surprising - the "infinitely longrange and infinitely weak interactions" route to an explicit, analytic (mean-field-like) theory is well known [15 18]. What is new here is the connection between nonintegrable interactions and the much-studied "nonextensive thermodynamics." This connection rests on an additional result, namely the demonstration that any ordering of the limits $L, R \rightarrow \infty$ yields the same free energy. For the limit $L \rightarrow \infty$ first, the free energy is obtained directly from a Kac-potential treatment (at least for $\sigma<0$; the case $\sigma=0$ is treated separately), while when the limit $R \rightarrow \infty$ is taken first, we obtain, as an intermediate step, a finite system with constant

*Permanent address.

${ }^{\dagger}$ The work described here has been initiated at the Institut für Physik, Johannes Gutenberg-Universität, Mainz, Germany. 
interactions proportional to $1 / L^{d}$-i.e., without approximation we obtain mean-field-like interactions! Finally, in the limit $R \propto L \rightarrow \infty$ the $R$-dependent prefactor multiplying the pair interactions may be regarded instead as a power of $L$. This reproduces all scaling results of nonextensive thermodynamics of which we are aware, reducing the study of these systems to the application of standard techniques with Boltzmann-Gibbs statistics. Furthermore, in the context of thermalized gravitational systems [19 21] our results provide what we believe is a new, direct connection to a rigorous Kac-potential treatment.

While a finite free-energy density is obtained in the $R, L \rightarrow \infty$ limit, the actual result depends explicitly on the cutoff function. To be specific, consider a fluid of density $\rho$ with the pair interaction

$$
u(r)= \begin{cases}\infty & r<a \\ -\frac{1}{R^{d-\tau}} \frac{w(r / R)}{r^{\tau}} & r>a, 0 \leq \tau<d \\ -\frac{1}{\ln R} \frac{w(r / R)}{r^{d}} & r>a, \tau=d\end{cases}
$$

where $\tau \equiv d+\sigma$ is introduced to avoid confusion with the negative values of $\sigma$, and where the cutoff function $w(x)$ - taken to be isotropic for simplicity-decays at least as fast as $1 / x^{d-\tau+\varepsilon}$ for positive $\varepsilon$, with $w(0)$ finite. The $R$-dependent prefactors are chosen to preempt the divergence of the energy in the limit $R \rightarrow \infty$. From this we obtain, in the limits $L, R \rightarrow \infty$, the Helmholtz free-energy density

$$
f(\rho, T)=\text { C.E. }\left\{f_{0}(\rho, T)-A \rho^{2}\right\},
$$

where C.E. $\{\ldots\}$ represents the maximal convex envelope, $f_{0}(\rho, T)$ is the hard-core free energy (which is strictly proportional to $T$ ), and

$$
A=\left\{\begin{array}{lc}
\frac{1}{2} S_{d} \int_{0}^{\infty} w(x) x^{d-\tau-1} d x & 0 \leq \tau<d \\
\frac{1}{2} S_{d} w(0) & \tau=d
\end{array} .\right.
$$

Here $S_{d}=2 \pi^{d / 2} / \Gamma(d / 2)$ is the surface area of a unit $d$ sphere. Up to a factor $-k_{B} T, A$ is just the second virial coefficient without the hard-core contribution.

The free energy thus obtained indeed depends explicitly on the cutoff function $w(x)$, at least for $\tau<d$, but does not depend on the system shape. We obtain quantitatively the same result for a lattice gas (hence also for Ising spin systems, cf. Ref. [22], §II E) with the substitution of the lattice hard-core free energy being the only modification. In this context, we recall that the first application of the Kac potential to spin systems is due to Baker [16].

The dependence on details of the cutoff regulator has important implications when this solution is recast in the interpretation of "nonextensivity." For the non-periodic case of nonextensive thermodynamics, where the finite system size is used to regulate the energy, the bulk quantities will depend on both boundary effects and the system shape, a point we have not found mentioned in previous studies. Furthermore, when periodic boundary conditions are employed and the interaction is cut off at some fraction of the system size, the bulk thermodynamics will depend on precisely which fraction is used, notwithstanding statements to the contrary [8] (cf. our discussion in Sec. IV below). The remainder of the paper is organized as follows. In Section II we present our mathematical treatment of the various limits which lead to the results above. Next we present briefly the critical properties of these systems in Section III. Since we can interpret our results in the language of nonextensive thermodynamics, this connection is presented and discussed in Section IV, and various difficulties with nonextensive thermodynamics are brought to light, including the above-mentioned system-shape dependent "bulk" thermodynamics. Finally, we conclude with a summary of our main results, and some remarks on the connection to work done in the area of gravitational systems.

\section{MATHEMATICAL TREATMENT}

\section{A. Thermodynamic limit with finite range}

We begin by considering a fluid in $d$ spatial dimensions with the pair-interaction potential given by (1), with finite $R$ and in the thermodynamic limit. Then for $0 \leq \tau<d$ our main results (2) and (3) for the limit $R \rightarrow \infty$ follow immediately from the rigorous treatment of Lebowitz and Penrose [18]. To see this, define

$$
\phi(x)=w(x) / x^{\tau},
$$

in which case (1) becomes $u(r>a)=-R^{-d} \phi(r / R)$, the canonical Kac potential, with $\phi(x)$ satisfying the necessary conditions for the proof given in [18], cf. Eqs. (1.21a)-(1.21c) in this reference.

It is also possible and useful (for the $\tau=d$ case) to understand this result from the Mayer cluster or virial expansion about a reference hard-core potential [22]. Specifically, the Mayer function $\tilde{f}(r)=e^{-\beta u(r)}-1$ (not to be confused with the free-energy density) may be decomposed as

$$
\tilde{f}(r)=[\theta(r-a)-1]+\theta(r-a)\left[e^{-\beta u(r)}-1\right],
$$

with $\theta(x)$ the Heaviside step function. The first squarebracket term is the Mayer function for the hard-core potential and the second term accounts for the attractions. Each irreducible cluster of the virial expansion may be replaced with a sum of clusters in which the individual bonds are replaced, in turn, with each of the hard-core and attraction bonds.

The hard-core bonds are independent of the cutoff $R$, so the sum of diagrams containing only these bonds yields the hard-core free energy (via Legendre transformation), 
which is unaffected by the limit $R \rightarrow \infty$. In what follows, we summarize the argument for why the attraction bond 2-cluster is the only other term which survives the $R \rightarrow \infty$ limit.

The attraction bonds go to $-\theta(r-a) \beta u(r) \propto 1 / R^{d-\tau}$ for large $R$ (with $\tau<d$, for now). Each vertex that is free to integrate over space gives a factor $\int d^{d} r w(r / R) / r^{\tau} \sim$ $R^{d-\tau}$. Consider first diagrams with only attraction bonds: the $n$th order ring diagram (each vertex having exactly two bonds) with $n \geq 3$ has $n$ bonds and $n-1$ free vertices, and so vanishes as $1 / R^{d-\tau}$ for large $R$. All other $n \geq 3$ clusters have a higher ratio of bonds to vertices, and so these also vanish when all bonds are attractive.

Replacing an attraction bond with a hard-core bond removes one factor of $1 / R^{d-\tau}$ but also kills one of the free vertex integrals, i.e. the integration region is constrained to the scale of the hard core. The net effect for ring diagrams is that they remain of the same order in $R$, and so vanish for $n \geq 3$. For more complicated irreducible clusters, a situation can arise where two vertices mutually constrained by hard-core bonds are also connected by an attraction bond. In this case, replacing the attraction bond with a hard-core bond does increase the order of the diagram by a factor $R^{d-\tau}$. However, this situation can only occur for diagrams with higher powers of $1 / R$ than the ring diagrams, and can never bring them up to order $R^{0}$. Consequently, all diagrams with $n \geq 3$ and at least one attraction bond vanish as $R \rightarrow \infty$.

The $n=2$ case is distinct as it has one bond and one free integral, so it provides an $R^{0}$ contribution

$$
\begin{aligned}
a_{2} & =\lim _{R \rightarrow \infty}-\frac{\beta}{2} \int_{r>a} d^{d} r \frac{w(r / R)}{R^{d-\tau} r^{\tau}} \\
& =\lim _{R \rightarrow \infty} \frac{-\beta S_{d}}{2} \int_{a / R}^{\infty} w(x) x^{d-\tau-1} d x \\
& =-\beta A,
\end{aligned}
$$

where $A$ is given in (3).

Thus the virial expansion reproduces (2), apart from the convex envelope. The failure of this otherwise exact method to reproduce the Maxwell construction is that the virial expansion relies on a homogeneous density and breaks down when this is not the case. Nevertheless, since it is exact whenever the density is homogeneous, the virial expansion supplemented with the second law (convexity) prescribes a unique free energy, and so can be regarded as providing the rigorous result.

The utility of the virial-expansion method is that it applies to the borderline case $\tau=d$, where we cannot directly map to the results of [18]. The hard-core terms are unmodified, attraction bonds now go as $1 / \ln R$, and free vertex integrals give

$$
\int_{r \gtrsim a} d^{d} r w(r / R) / r^{d} \sim \ln R
$$

The sum of diagrams with $n \geq 3$ and at least one attraction bond is $O(1 / \ln R$ ) (provided there are no surviving resummations of $1 /(\ln R)^{n}$ terms $)$. The $n=2$ attraction bond diagram gives the only remaining contribution

$$
\begin{aligned}
a_{2} & =\lim _{R \rightarrow \infty} \frac{-\beta}{2} \int_{r>a} d^{d} r u(r) \\
& =\lim _{R \rightarrow \infty} \frac{-\beta S_{d}}{2 \ln R} \int_{a / R}^{\infty} w(x) x^{-1} d x \\
& =-\frac{1}{2} \beta S_{d} w(0)
\end{aligned}
$$

which again leads to (2) and (3). Interestingly, the bulk thermodynamics in this marginal case is not sensitive to the details of the cutoff function.

\section{B. Infinite range with finite system size}

Now consider the same fluid system, but taking $R \rightarrow$ $\infty$ with finite $L$. For clarity, we begin by considering a one-dimensional system with $0 \leq \tau<1$, in which the periodic boundary conditions lead to the effective pair potential $(r>a)$

$$
u_{\mathrm{eff}}(r)=-\frac{1}{R^{1-\tau}} \sum_{n=-\infty}^{\infty} \frac{w(|n L+r| / R)}{|n L+r|^{\tau}}
$$

where $r$ is understood to be less than $L / 2$. As $R$ becomes large, the direct $(n=0)$ interaction becomes negligible and an increasingly large number of terms contribute to the sum. By use of the expansions

$$
|n L+r|^{-\tau}=|n L|^{-\tau}\left(1-\frac{\tau r}{n L}+\frac{\tau(\tau+1) r^{2}}{2 n^{2} L^{2}}-\ldots\right)
$$

and

$$
\begin{aligned}
w\left(\frac{|n L+r|}{R}\right)= & w(|n| L / R)+\operatorname{sign}(n) \frac{r}{R} w^{\prime}(|n| L / R) \\
& +\frac{r^{2}}{2 R^{2}} w^{\prime \prime}(|n| L / R) \pm \ldots
\end{aligned}
$$

where we assume $w(x)$ to be analytic, we may rewrite $u_{\text {eff }}(r)$ for large $R$ as

$$
\begin{aligned}
u_{\text {eff }}(r)= & -\frac{2}{R} \sum_{n=1}^{\infty}\left(\frac{R}{n L}\right)^{\tau}\left\{w\left(\frac{n L}{R}\right)\left[1+\mathcal{O}\left(\frac{1}{n^{2}}\right)\right]\right. \\
& \left.+\mathcal{O}\left(\frac{L}{n R}, \frac{L^{2}}{R^{2}}\right)\right\} .
\end{aligned}
$$

The convergence of this series is guaranteed by the shape of the cutoff function $w$ and its derivatives. Note that the leading sum for large $R$ is independent of the spatial separation $r$; the correction terms are down by a factor $(L / R)^{1-\tau}$. In the limit of large $R$, we may, in turn, express this sum as an integral 


$$
\begin{aligned}
\lim _{R \rightarrow \infty} u_{\mathrm{eff}}(r) & =-\frac{2}{R} \int_{0}^{\infty}(R / n L)^{\tau} w(n L / R) d n \\
& =-\frac{2}{L} \int_{0}^{\infty} w(x) x^{-\tau} d x .
\end{aligned}
$$

For the borderline case $\tau=1$, Eqs. (9) and (12) have to be multiplied by $(\ln R)^{-1}$. Furthermore Eq. (13) now requires a lower integration limit $n=1$ (or any constant) as a regulator. The pair interaction is then

$$
\begin{aligned}
u_{\mathrm{eff}}(r) & =-\frac{2}{L \ln R} \int_{L / R}^{\infty} w(x) x^{-1} d x \\
& =-\frac{2}{L \ln R}\left[w(0) \ln R+O\left(R^{0}\right)\right] .
\end{aligned}
$$

In the limit $R \rightarrow \infty$, we find the effective pair potential is again independent of the spatial separation, $u_{\text {eff }}(r)=-2 L^{-1} w(0)$.

The generalization of this treatment to higher dimensionalities is straightforward. For a $d$-dimensional system of size $L_{1} \times L_{2} \times \cdots \times L_{d}$, with periodic boundary conditions and $0 \leq \tau<d$, the effective pair potential $(r>a)$ is

$$
u_{\mathrm{eff}}(r)=-\frac{1}{R^{d-\tau}} \sum_{n_{1}=-\infty}^{\infty} \cdots \sum_{n_{d}=-\infty}^{\infty} \frac{w\left(\frac{\tilde{r}\left(n_{1}, \ldots, n_{d}\right)}{R}\right)}{\tilde{r}\left(n_{1}, \ldots, n_{d}\right)^{\tau}}
$$

where

$$
\tilde{r}\left(n_{1}, \ldots, n_{d}\right) \equiv\left|\mathbf{r}+n_{1} L_{1} \hat{\mathbf{x}}_{1}+\ldots+n_{d} L_{d} \hat{\mathbf{x}}_{d}\right|
$$

is the separation corresponding to the $\left(n_{1}, \ldots, n_{d}\right)$ periodic repeat, and the $\hat{\mathbf{x}}_{i}$ are orthonormal vectors. In the limit of infinite interaction range $R$, the direct interaction term (all $n_{i}=0$ ) becomes negligible compared to the sum and we find the following generalization of Eq. (13):

$$
u_{\mathrm{eff}}(r)=-\frac{S_{d}}{V} \int_{0}^{\infty} w(x) x^{d-1-\tau} d x,
$$

where $S_{d}$ has been introduced below Eq. (3) and $V \equiv$ $\prod_{i=1}^{d} L_{i}$. We note that the strength of this constant interaction is inversely proportional to the volume, just as one expects for a mean-field-like system [23]. For $\tau=d$ one has, in the limit $R \rightarrow \infty, u_{\text {eff }}(r)=-S_{d} V^{-1} w(0)$. Since a constant pair interaction gives a configurationindependent energy density $\frac{1}{2} u_{\text {eff }} \rho^{2} V$, the energy and entropy contributions to the free energy decouple, and one obtains (2) directly in the thermodynamic limit.

Instead of a "soft" cutoff provided by the (analytic) function $w(x)$, one can also introduce a "hard" cutoff at a distance $R$, which is equivalent to taking, say, $w(x)=\theta(1-x)$ in Eq. (1). We start again with $d=1$ and $\tau<d$. The effective pair potential can be written as

$$
u_{\mathrm{eff}}(r)=-\frac{1}{R^{1-\tau}} \sum_{n=-R / L}^{R / L} \frac{1}{|n L+r|^{\tau}}
$$

where, for simplicity, we have taken $R$ to be an integer multiple of $L$. For large $R$, the $n=0$ term becomes negligible compared to the sum and by use of the expansion (10) we rewrite $u_{\text {eff }}(r)$ as

$$
u_{\mathrm{eff}}(r)=-\frac{2}{L}\left(\frac{R}{L}\right)^{\tau-1} \sum_{n=1}^{R / L}\left\{\frac{1}{n^{\tau}}\left[1+\mathcal{O}\left(\frac{1}{n^{2}}\right)\right]\right\} .
$$

The $\mathcal{O}\left(n^{-2}\right)$ correction terms are again down by a factor $(R / L)^{\tau-1}$ from to the leading sum [compare to (12)]. As before, we end up with a constant effective interaction $\lim _{R \rightarrow \infty} u_{\text {eff }}(r)=-2 L^{-1} /(1-\tau)$. For $\tau=1$, the correction terms decay only like $1 / \ln R$ and $\lim _{R \rightarrow \infty} u_{\text {eff }}(r)=-2 / L$. Since the case of general dimensionality can be treated along the same lines, we only mention the resulting values for the constant pair potential: $\lim _{R \rightarrow \infty} u_{\text {eff }}(r)=-S_{d} V^{-1} /(d-\tau)$ for $\tau<d$ and $\lim _{R \rightarrow \infty} u_{\text {eff }}(r)=-S_{d} / V$ for $\tau=d$. These results are all consistent with (2) with $w(x)=\theta(1-x)$.

Thus, we have shown that, in the limit of infinite interaction range, all pair interactions are identical even in a finite system, for any $0 \leq \tau \leq d$. For $\tau>d$ on the other hand, the sum in Eqs. (9), (17) is convergent and hence the $R$-dependent prefactor in $u(r)$ should be omitted. The first term within the square brackets is then no longer negligible in the limit $R \rightarrow \infty$, which leads to an effective interaction that depends both on $r$ and on $\tau$ and consequently to nontrivial critical behavior. Interestingly, the critical exponents retain their classical values until $\tau>3 d / 2$, except for the correlation-length exponent $\nu$ and the correlation-function exponent $\eta$ [1]. The critical temperature and other nonuniversal quantities exhibit a nontrivial $\tau$ dependence already for $\tau>d$, cf. Ref. [24] and references therein.

\section{Lattice gas}

So far we have only discussed continuum fluid criticality. The generalization to a lattice gas (and, of course, to the Ising ferromagnet) results in the same free energy (2) with the appropriate lattice hard core $f_{0}(\rho)$. This may be seen most directly in the $R \rightarrow \infty$ first case, where the pair interactions go to a constant. This derivation applies both for particles in a continuum or on a lattice (indeed, no specification was made), and so the constant interactions are obtained in both cases. The final step of constructing the free energy from the decoupled energy and entropy reveals that the lattice hard-core free energy is the appropriate one to use in (2).

For the $L \rightarrow \infty$ first limit, one must use a lattice generalization of the Mayer expansion. Such an expansion would give for non-interacting particles the lattice hardcore free energy. The lattice sums for clusters with attraction bonds can be taken to integrals in the large $R$ limit, and so the rest of the continuum derivation applies. 


\section{Thermodynamic limit with a system-size dependent range}

The final case we consider is that of $R \propto L \rightarrow \infty$, the case most directly applicable to "nonextensive thermodynamics." Consider a finite system with periodic boundary conditions (with all dimensions $L_{i} \propto L$ ) and direct pair interactions given by (11) for some finite $R$. The periodic boundary conditions give rise to the effective pair potential (17). The virial expansion for the pressure may then be obtained from a finite-volume cluster expansion with the Mayer function $\tilde{f}=\exp \left(-\beta u_{\text {eff }}\right)-1 \sim-\beta u_{\text {eff }}$ for large $R$. Since the effective interaction is a sum of pair interactions, a simple $u_{\text {eff }}$ cluster such as the irreducible 3-cluster shown in Fig. 11(a) decomposes into a large number of pair-interaction bond clusters [cf. Fig. 11(b)]. Note that, in order to avoid overcounting, one end of every bond must remain in the original finite volume, but the other end may be taken to lie in any of the periodic repeats (and these still contribute to the virial expansion as they are parts of an irreducible cluster).

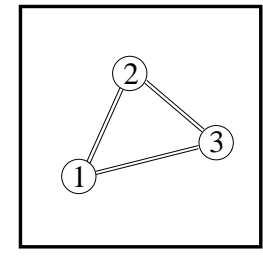

(a)

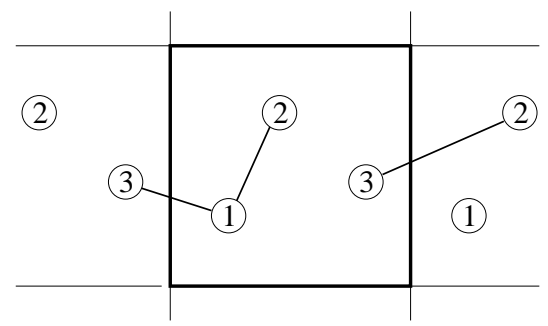

(b)
FIG. 1. (a) The 3-cluster formed with the effective interaction $u_{\text {eff }}$ and (b) one contribution to the decomposition of this cluster into pair interactions $u(r)$.

In spite of these complications, we remark that all hard-core bonds are the same as in the previous $(L \rightarrow \infty$ first) case, since they only appear in the direct interaction of the original pair. Hence the hard-core bonds sum to the hard-core free energy regardless of whether $L$ and $R$ are simultaneously taken to infinity, or $L$ first.

Furthermore, all $n$-clusters with $n \geq 3$ and at least one attraction bond can be shown to still vanish as $R, L \rightarrow \infty$. First, all bonds still carry a factor of $1 / R^{d-\tau}$ for large $R$, as before. Free vertices integrate to

$$
\int_{L^{d}} d^{d} r r^{-\tau} w(r / R) \sim R^{d-\tau} \int_{(L / R)^{d}} d^{d} x x^{-\tau} w(x)
$$

for the direct interaction of the original pair, which goes as $R^{d-\tau}$ times a finite factor for large $R$. For a pair interaction involving a neighboring replica, the vertex integration is the same as above with a shift in the argument of $w(\tilde{r} / R)$, where $\tilde{r}$ [see Eq. (18)] goes roughly as $r+c L$. The resulting vertex integration will also scale as $R^{d-\tau}$, provided $r+c L \lesssim R$. Since $\tilde{r}$ increases for each increasingly remote replica, the cutoff function $w(x)$ will ensure that only a number $\mathcal{O}\left((R / L)^{d}\right)$ of such bonds will contribute. Hence free vertex integration with effective interaction bonds, while considerably more complicated, still results in a factor $R^{d-\tau}$ at large $R$. Previous arguments from Section II A then apply, and so these terms all vanish when $R, L \rightarrow \infty$.

The remaining 2-cluster integral may be written as

$$
\begin{aligned}
a_{2} & =\lim _{R \rightarrow \infty} \frac{\beta}{2} \int_{r>a} d^{d} r u_{\mathrm{eff}}(r) \\
& \sim \frac{-\beta}{2 R^{d-\tau}} \prod_{i=1}^{d}\left(\sum_{n_{i}=-\infty}^{\infty} \int_{0}^{L_{i}} d x_{i}\right) \frac{w(\tilde{r} / R)}{\tilde{r}^{\tau}} \\
& =\frac{-\beta}{2 R^{d-\tau}} \prod_{i=1}^{d}\left(\sum_{n_{i}=-\infty}^{\infty} \int_{n_{i} L_{i}}^{\left(n_{i}+1\right) L_{i}} d x_{i}\right) \frac{w(r / R)}{r^{\tau}}
\end{aligned}
$$

where the sums cover all replicas (and we have omitted writing the hard-core condition for clarity). In going from (24) to (25) the integrand variable changed from $\tilde{r}$ to $r$, consistent with the definition of $\tilde{r}$. The remaining integrals piece together a single volume integral over all space, so

$$
\begin{aligned}
a_{2} & =\lim _{R \rightarrow \infty} \frac{-\beta}{2 R^{d-\tau}} \int_{r>a} d^{d} r w(r / R) / r^{\tau} \\
& =-\beta A,
\end{aligned}
$$

following (6). Combining this with the hard-core contribution gives the same free energy (2) in the $R, L \rightarrow \infty$ limit as was found previously for $L$ or $R$ going to infinity first. The significance of this simultaneous limit towards nonextensive thermodynamics will be discussed in Section IV.

\section{CRITICAL BEHAVIOR}

The critical properties of nonintegrable systems are readily found from the Helmholtz free-energy density (2) via standard procedures. For example, the critical density can be obtained from $\partial^{3} f(\rho, T) /\left.\partial \rho^{3}\right|_{\rho_{c}}=0$, which reduces to the temperature (and attraction) independent condition $\partial^{3} f_{0}(\rho, T) /\left.\partial \rho^{3}\right|_{\rho_{c}}=0$. The critical temperature is then found from $\partial^{2} f\left(\rho, T_{c}\right) /\left.\partial \rho^{2}\right|_{\rho_{c}}=0$ which gives

$$
k_{B} T_{c}=2 A\left(\frac{\partial^{2}\left(\beta f_{0}\right)}{\partial \rho^{2}}\right)_{\rho=\rho_{c}}^{-1} .
$$

For a hypercubic lattice with lattice constant $a$, the lattice-gas hard-core free energy is $\beta f_{0}^{\mathrm{LG}}=\rho \ln \rho+\left(a^{-d}-\right.$ $\rho) \ln \left(1-a^{d} \rho\right)$. This results in the critical values

$$
\text { lattice gas: } \quad a^{d} \rho_{c}=1 / 2 \quad k_{B} T_{c}=A /\left(2 a^{d}\right) .
$$


Although the free energy for continuum hard spheres (diameter $a$ ) is not known exactly, a very good approximation in three dimensions is nevertheless given by the Carnahan-Starling (CS) expression

$$
\beta f_{0}^{\mathrm{CS}}(\rho)=\beta f^{\mathrm{Id}}(\rho)+\frac{\rho^{2} v_{0}\left(4-3 \rho v_{0}\right)}{\left(1-\rho v_{0}\right)^{2}},
$$

with $v_{0}=\pi a^{3} / 6$ the volume of the hard sphere and $f^{\text {Id }}$ the ideal gas free energy density. Using this for $f_{0}$ yields a quintic equation for $\rho_{c}$ with a unique positive root 25] and the critical values

$$
\mathrm{CS}: \quad a^{3} \rho_{c} \simeq 0.249129 \quad k_{B} T_{c} \simeq 0.180155 A / a^{3} .
$$

The susceptibility $\chi$, defined as the ratio of the isothermal compressibility to the ideal gas compressibility,

$$
\chi^{-1}(\rho, T) \equiv \rho \frac{\partial^{2}(\beta f)}{\partial \rho^{2}},
$$

exhibits near criticality the classical divergence $\chi \sim C_{+} / t$ for $\rho=\rho_{c}$ and positive reduced temperature $t \equiv(T-$ $\left.T_{c}\right) / T_{c}$. From (2) we find

$$
C_{+}=\left(\left.\rho_{c} \frac{\partial^{2}\left(\beta f_{0}\right)}{\partial \rho^{2}}\right|_{\rho_{c}}\right)^{-1},
$$

which results in $C_{+}^{\mathrm{LG}}=1 / 2$ for the lattice gas and $C_{+}^{\mathrm{CS}} \simeq 0.361569$ for the $d=3$ hard-sphere fluid, approximated by the CS expression.

Finally, the order parameter for $T<T_{c}$ is given by

$$
a^{d}\left|\rho-\rho_{c}\right|=B \sqrt{-t}
$$

for $t$ sufficiently small, with

$$
B=a^{d}\left(\frac{18\left[\partial^{2} f_{0} / \partial \rho^{2}\right]_{\rho_{c}}}{\left[\partial^{4} f_{0} / \partial \rho^{4}\right]_{\rho_{c}}}\right)^{1 / 2} .
$$

This evaluates to $B^{\mathrm{LG}}=3 / 2$ and $B^{\mathrm{CS}} \simeq 1.13459$.

\section{NONEXTENSIVE THERMODYNAMICS}

As indicated in the Introduction, the main motivation for this work stems from the considerable attention systems with nonintegrable interactions have received in the context of "nonextensive thermodynamics." An essential aspect of these studies of nonextensivity is the use of the system size as the regulator for the energy. Furthermore, the interactions are not scaled by a negative power of the system size but left with strength of order unity.

Our cutoff interaction with range $R$ can be interpreted directly in terms of this nonextensive thermodynamics for $R / L$ constant, which reproduces the system-size regulator. The negative power of $L$ multiplying our pair interaction is eliminated by scaling the temperature according to $T \rightarrow L^{d-\tau} T$ in the Boltzmann factor $(T \rightarrow T \ln L$ for $\tau=d)$. The scaling of this system-size dependent "temperature" (and consequently the free energy) matches exactly the conjectures of nonextensive thermodynamics, thus we have derived rigorously the primary conclusions of Refs. [9, 8, 11, 12] using only standard methods. We note that, despite what has been suggested in Ref. [26], neither the explicit free energy nor exact results for the nature of the criticality were obtained in Refs. [9,11].

Next we want to discuss some examples from the recent literature and point out a few problems attached to the interpretation of nonextensive thermodynamics. First, a pervasive notational problem in the nonextensive thermodynamics literature is the use of "long-range interactions" to mean "nonintegrable interactions." The former term already has a standard meaning within the considerably more important class of integrable interactions [27,28,1]. The converse problem also exists, where all integrable interactions (including the true long-range interactions) are termed "short-range" (see, e.g., Ref. [29]).

Many papers addressing nonintegrable interactions have relied on numerical simulations of finite systems. In general, the required regularization of the interactions is carried out either by imposing free boundary conditions (which leads to an inhomogeneous system) or by adopting periodic boundary conditions and cutting off the interaction at half the system size, being the maximum separation between the particles. For example, Curilef and Tsallis [8] have performed molecular-dynamics simulations of fluids in $d=2,3$ dimensions with LennardJones-like interactions, with an attractive tail decaying like $r^{-\tau}$ and $1 \leq \tau \leq 2 d$. It is explicitly stated (Ref. [8], p. 271) that, in the thermodynamic limit, no physical consequences emerge from the ("computationally convenient") adoption of a cutoff at half the size of the simulation box. Our exact solution presented in Sec. II shows that this is not correct for $\tau<d$, but that rather different bulk thermodynamics emerges for different cutoff distances and cutoff functions.

In Ref. [29], rings of magnetic particles in a colloidal suspension have been studied numerically, where the nonmagnetic part of the interactions has the abovementioned generalized Lennard-Jones form. The main results are heuristically interpreted in terms of "nonextensive thermodynamics" by observing that the size dependence of the total energy of the rings can be well described by a scaling law obtained from the integrated interaction (which is essentially a mean-field-like approximation). This scaling law is just what is also found within the " $q$-generalized thermodynamics" of Ref. [6], commonly referred to as Tsallis $q$-statistics, and consequently a $\tau$ dependence $(\tau=d+\sigma)$ is proposed for the so-called "nonextensivity parameter" $q$ appearing in this formalism: $q=1$ (corresponding to Boltzmann-Gibbs statistics) for $\tau>d$ and $q=2-d / \tau$ for $\tau \leq d$. Also in Ref. 30 it has been conjectured that $q$ is a $d$ - and $\tau$-dependent quantity for $\tau \leq d$. It appears that these 
conclusions have been since revised [10,31, and these systems are now classified as "weakly violating" BoltzmannGibbs statistics, meaning that $q=1$, whereas several thermodynamic quantities lose their extensivity. Nevertheless, recent studies have continued to explore $q \neq 1$ values for $\tau \leq d$, citing an alleged natural connection between Tsallis statistics and nonintegrable interactions [32]. Our results show that the same energy scaling for $\tau \leq d$ may be obtained with the conventional value $q=1$.

Inspired by the system-size dependence of the energy found in Ref. 229], an Ising model with interactions decaying as $r^{-\tau}(\tau \geq 0)$ and free boundary conditions has been analyzed by mean-field methods in Ref. [9]. On the basis of the resulting values of the critical temperature for $\tau=0$ and $\tau=d$, it was then conjectured that the meanfield prediction for the critical temperature might hold for all $0 \leq \tau \leq d$. Our exact result now demonstrates that this is indeed true, but reveals in addition an awkward consequence of the boundary conditions adopted in Ref. [9]: since the thermodynamic limit explicitly depends on the choice of the cutoff function, an inhomogeneous system with an inhomogeneous cutoff will lead to bulk thermodynamics that depends on the shape of the system, which to our judgment constitutes an undesirable feature of the nonextensivity formulation. We note that already in an early study of the Ising model with longrange interactions [33] a system-shape dependence of the thermodynamic properties has been observed for the case of conditionally convergent lattice sums with $\tau=d$ (such as dipolar forces). Also the exactness of mean-field theory for shape-independent forces in the limit $\tau \rightarrow d+$ has been obtained in the same reference, essentially from an observation similar to ours for general $0 \leq \tau \leq d$, namely that one divergent term dominates all other terms in the lattice sum. However, for the case $\tau<d$, we have been unable to find in the literature any mention of the systemshape dependent thermodynamics that must result for all inhomogeneous systems with nonintegrable interactions and a system-size dependent cutoff.

\section{SUMMARY AND CONCLUSIONS}

In summary, we have shown that $d$-dimensional, periodic systems with nonintegrable, algebraically decaying interactions, i.e., interactions of the form $u(r) \sim-1 / r^{\tau}$, with $0 \leq \tau \leq d$, are exactly described by mean-field theory, upon introduction of a cutoff $R$ in the interaction range and the proper $R$-dependent rescaling of the interaction strength. This proof holds for either order of limits $R \rightarrow \infty$ and $L \rightarrow \infty$ (where $L$ is the linear system size), including the simultaneous limit, and the resulting free energy depends on the details of the cutoff.

Our study employs Boltzmann-Gibbs statistics and pertains directly to nonextensive thermodynamics, providing explicit, exact results for the thermodynamics and critical behavior. In doing so, we show that nonintegrable interactions do not require 30,32] the application of generalized $q$-statistics. Furthermore, the explicit regulator dependence - cutoff length, cutoff shape, and even system shape for inhomogeneous systems - is demonstrated, a topic which has been mostly neglected in nonextensivity studies.

On an intuitive level, our findings for the case of finite systems with infinite $R$ (Section II B) result from the divergence of the lattice sums over the periodic copies of the system under consideration; these divergent sums then dominate the direct pair interaction, and are, to leading order, independent of the spatial separation between particles. A suitable normalization is indispensable for the existence of the thermodynamic limit and - as we have pointed out - a regulator depending on the number of interactions rather than on the system size emerges as the natural choice. The resulting effective pair interaction is then independent of the spatial separation, for $0 \leq \tau \leq d$.

We have concentrated on both fluids and lattice gases. However, since our method for the $R \rightarrow \infty$ first case demonstrated how the effective interaction becomes independent of spatial separation, our results immediately carry over to large classes of other systems as well (obviously, the critical properties obtained in Sec. [II] explicitly refer to systems with a one-component order parameter). These include general $\mathrm{O}(n)$ models $(X Y$, Heisenberg, $\ldots$ ) and Potts models. In this context we note that in earlier work the exactness of mean-field theory in the limit $\tau \rightarrow d+$ has been found not only for the Ising model $(n=1)$ 33] but also for the Husimi-Temperley mean spherical model $(n \rightarrow \infty)$ 34.

Our explicit result of an analytic free energy, generalized to the systems mentioned above, explains a number of numerical results obtained for systems with nonintegrable interactions. This includes the moleculardynamics simulations of systems with a generalized Lennard-Jones potential discussed in Sec. IV] 77, \&], Monte Carlo simulations of one-dimensional Ising [9], Potts [11], and $X Y$ [12] models with $\tau<1$, and the scaling properties found in Refs. [29, 10, 35, 13]. In Ref. [36], a subleading term in the spin-spin correlation function of the nonintegrable Ising chain was considered and on the basis of Monte Carlo simulations of finite systems with $\tau=0.50$ and $\tau=0.75$ it was concluded that these correlations are correctly described by mean-field theory.

Finally, we remark on the connection of these results to gravitational systems, where $d=3$ and $\tau=1$. Since the masses of the particles and the gravitational coupling are presumably fixed, we are not at liberty to scale to infinitely weak interactions. However, following the "nonextensive thermodynamics" formulations, we can consider $R \propto L$ for a finite system and regard the prefactor in the pair interactions as belonging to a rescaled temperature $T \rightarrow L^{d-\tau} T=L^{2} T$. At the same time, we note that such a divergent temperature appears of limited practical use.

The existence of a phase transition in these systems has been studied for fermionic particles in the 
context of Thomas-Fermi theory [19,20]. Most studies have considered gravitationally interacting particles in a non-periodic, finite-sized system, concentrating on the asymptotic, large- $N$ limit. Here they obtain nonextensivity in the particle number $N$, with the energy per particle growing as $N^{4 / 3}$ (in contrast to $N^{0}$ for integrable interactions). In Ref. [21] the energy densities were suitably rescaled to enable the infinite-volume limit, which presumably comes closest to what has been presented in the current work.

However, these studies differ from ours due to the fermionic character of the particles, which is used to regulate the short-distance behavior. Classical gravitational systems have previously been studied as well [37, 38, again in a non-periodic, finite-sized box, with various forms of short-distance regulators. In this case the energy per particle scales as $N$, essentially because the pair interaction, while decaying as $1 / r$, has a minimum value for all particles that is proportional to $1 / L$, due to the fixed system size. In contrast, our thermodynamic limit, $L \rightarrow \infty$ with fixed particle density, combined with the long-distance power-law tails of the pair potential, implies an energy per particle growing as $N^{2 / 3}$ (or $N^{1-\tau / d}$ for general $\tau<d$ ). Finally, we mention Ref. [39] for a recent review of some interesting features of thermalized gravitational systems.

\section{ACKNOWLEDGMENTS}

We wish to thank Professor Kurt Binder for a stimulating remark, and Professor Michael E. Fisher and Professor Bob Dorfman for helpful comments on the manuscript. We are indebted to Professor Michael Kiessling for enlightening us on the connection to gravitational systems. The hospitality of the Condensed Matter Theory Group at the Johannes Gutenberg-Universität Mainz, where this work was initiated, is gratefully acknowledged. B.P.V.-L. acknowledges support from Sonderforschungsbereich 262. E.L. acknowledges financial support through a fellowship of the Max-Planck-Institute for Polymer Research, from the National Science Foundation (through Grant No. CHE 99-81772 to Prof. M.E. Fisher), and from the Department of Energy, Office of Basic Energy Sciences (through Grant No. DE-FG0298ER14858 to Prof. A.Z. Panagiotopoulos).

[1] M. E. Fisher, S.-k. Ma, and B. G. Nickel, Phys. Rev. Lett. 29, 917 (1972).

[2] J. Sak, Phys. Rev. B 8, 281 (1973).

[3] D. Ruelle, Helv. Phys. Acta 36, 183 (1963).
[4] M. E. Fisher and D. Ruelle, J. Math. Phys. 7, 260 (1966).

[5] M. E. Fisher and J. L. Lebowitz, Comm. Math. Phys. 19, 251 (1970).

[6] C. Tsallis, J. Stat. Phys. 52, 479 (1988).

[7] J. R. Grigera, Phys. Lett. A 217, 47 (1996).

[8] S. Curilef and C. Tsallis, Phys. Lett. A 264, 270 (1999).

[9] S. A. Cannas and F. A. Tamarit, Phys. Rev. B 54, 12661 (1996).

[10] L. C. Sampaio, M. P. de Albuquerque, and F. S. de Menezes, Phys. Rev. B 55, 5611 (1997).

[11] S. A. Cannas, A. C. N. de Magalhães, and F. A. Tamarit, Phys. Rev. B 61, 11521 (2000).

[12] F. Tamarit and C. Anteneodo, Phys. Rev. Lett. 84, 208 (2000).

[13] S. A. Cannas, Physica A 358, 32 (1998).

[14] B. P. Vollmayr-Lee and Erik Luijten, Phys. Rev. Lett. 85, 470 (2000).

[15] M. Kac, Phys. Fluids 2, 8 (1959).

[16] G. A. Baker, Phys. Rev. 122, 1477 (1961).

[17] M. Kac, G. E. Uhlenbeck and P.C. Hemmer, J. Math. Phys. 4, 216 (1963).

[18] J. L. Lebowitz and O. Penrose, J. Math. Phys. 7, 98 (1966).

[19] W. Thirring, Lehrbuch der Mathematischen Physik, Vol. 4 (Springer, Vienna, 1980).

[20] J. Messer, Temperature Dependent Thomas-Fermi Theory (Springer, Berlin, 1981).

[21] A. Pflug, Comm. Math. Phys. 78, 83 (1980).

[22] P. C. Hemmer and J. L. Lebowitz, in Phase Transitions and Critical Phenomena, Vol. 5B, edited by C. Domb and M. S. Green (Academic, London, 1976).

[23] R. J. Baxter, Exactly Solved Models in Statistical Mechanics (Academic, London, 1982).

[24] E. Luijten and H. W. J. Blöte, Phys. Rev. B 56, 8945 (1997).

[25] M. E. Fisher and B. P. Lee, Phys. Rev. Lett. 77, 3561 (1996).

[26] R. Salazar and R. Toral, Phys. Rev. Lett. 85, 471 (2000).

[27] D. Ruelle, Comm. Math. Phys. 9, 267 (1968).

[28] F. J. Dyson, Comm. Math. Phys. 12, 91 (1969).

[29] P. Jund, S. G. Kim, and C. Tsallis, Phys. Rev. B 52, 50 (1995).

[30] F. D. Nobre and C. Tsallis, Physica A 213, 337 (1995).

[31] C. Tsallis, in Nonextensive Statistical Mechanics and Thermodynamics, edited by S. R. A. Salinas and C. Tsallis, Braz. J. Phys. 29, 1 (1999).

[32] R. Salazar and R. Toral, Phys. Rev. Lett. 83, 4233 (1999). Note also Ref. [26], which clarifies the boundary conditions employed.

[33] B. J. Hiley and G. S. Joyce, Proc. Phys. Soc. 85, 493 (1965).

[34] J. G. Brankov, Physica A 168, 1035 (1990).

[35] C. Anteneodo and C. Tsallis, Phys. Rev. Lett. 80, 5313 (1998).

[36] B. Bergersen, Z. Rácz, and H.-J. Xu, Phys. Rev. E 52, 6031 (1995).

[37] J. Messer and H. Spohn, J. Stat. Phys. 29, 561 (1982).

[38] M. K.-H. Kiessling, J. Stat. Phys. 55, 203 (1989).

[39] D. Lynden-Bell, Physica A 263, 293 (1999). 